\title{
Shedding of oocysts of Cryptosporidium in immunocompetent patients
}

\author{
R CAMERON SHEPHERD, CAROLE L REED, G P SINHA
}

\section{From the Royal Alexandra Hospital, Paisley, Scotland}

SUMMARY Forty nine patients (19 adults and 30 children) with oocysts of Cryptosporidium in their faeces had repeated stool specimens taken until oocysts could no longer be identified. They were found in the stools up to 35 days after the onset of symptoms in one patient, but most had stopped shedding them by 20 days. In 25 of the 49 patients in whom symptoms could be compared with the shedding of oocysts, $19(76 \%)$ had symptoms corresponding to the shedding period while symptoms persisted in four (16\%) after shedding had stopped.

Reports from many parts of the world have established the importance of the coccidian parasite Cryptosporidium as a gastrointestinal pathogen in man. ${ }^{1}$ We have found that in children in the west of Scotland it is the commonest cause of gastrointestinal symptoms after viruses, and in adults it ranks third after Salmonella and Campylobacter infections. ${ }^{2}$ It infects otherwise healthy individuals causing symptoms of variable severity; there is usually a violent attack of diarrhoea with abdominal pain, vomiting, and fever. The illness is self limiting and symptoms may be shortlived or persist for many weeks. ${ }^{3}$ In immunocompromised subjects the illness is prolonged, debilitating, and often fatal. ${ }^{4}$

The parasite Cryptosporidium seems to be a single species common to all the host organisms it infects. ${ }^{5}$ It is enzootic and spread is probably both from animals to man and from person to person by the faecal oral route. ${ }^{\prime}$ The life cycle takes place in the gut of the host and has been well documented by Bird and Smith. ${ }^{6}$ At the oocyst stage the organism is attached to enterocytes in the small and large intestine. Sporulation takes place in situ and "sporocysts" are shed into the faeces where they can be identified as so called "oocysts". The finding of these oocysts in the stool is generally taken to represent infection and infectivity. Estimates of the duration of excretion of the oocysts of Cryptosporidium vary widely. Current et al reported the shedding of oocysts up to one week after the onset of symptoms ${ }^{7}$ but Hunt et al found that $44 \%$ of their patients were excreting oocysts in the stools two weeks after the onset of symptoms. ${ }^{8}$ In a study of eight subjects Stehr-Green et al found that the duration of oocyst shedding ranged from eight to 50 days. ${ }^{9}$

Accepted for publication 21 April 1988
The duration of shedding of oocysts is important for $\vec{\overrightarrow{ }}$ clinical and public health reasons as it represents 0 the period of infectivity of the patient for crypto- $?$ sporidiosis. We have studied the incidence of crypto- $\vec{O}$ sporidiosis in the west of Scotland by investigating every case identified by a single department of $\frac{0}{\circ}$ microbiology serving a population of 270000 over a $\stackrel{\mathbb{S}}{-}$ two year period. Clinical and epidemiological data $\overrightarrow{0}$ have been reported elsewhere. ${ }^{2}$ We now report on the $\infty^{\circ}$ shedding of oocysts in the stools of our patients. We believe this to be the largest series of patients follow up until their stools were clear of oocysts.

\section{Patients and methods}

All specimens of stool submitted to the laboratory were examined for oocysts of Cryptosporidium on a faecal smear fixed with methanol and stained by a modified Ziehl Neelsen method using strong carbol fuchsin at room temperature. ${ }^{10}$ Specimens were submitted by general practitioners from the community, and also from hospital inpatients. As part of our study a visit to the household of each reported case was included in the routine duties of one of us (CLR) an infection control sister. Details of history and contacts were recorded and follow up specimens of stool were $\frac{D}{2}$ taken until oocysts had cleared completely. Further information was obtained from a questionnaire sent to $N$ the mothers of the 30 children in the study.

\section{Results}

During the two years January 1986 to December 1987

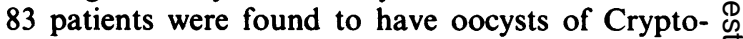
sporidium in their specimens of stool. The clinical details of these patients have been reported. ${ }^{2}$ For 
Days to last Day to last postive

negative

Children $\bullet$

Adults

2
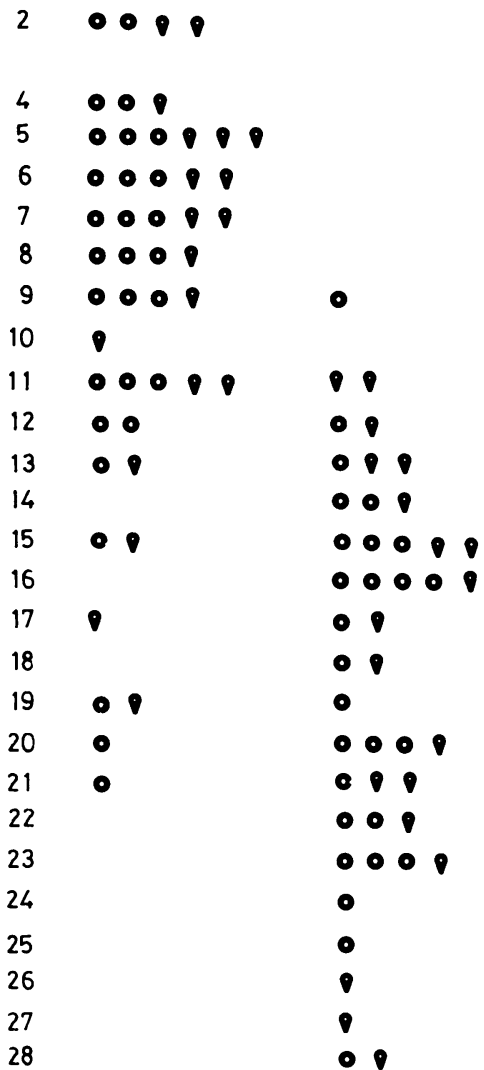

33

35

37

50

of the adults was 17-88 years, and of the children nine months-14 years. All patients were previously healthy and none was immunocompromised. The 49 patients provided a total of 178 stool specimens for examination, an average of three to four stools/patient.

The duration of shedding of oocysts from the onset of symptoms is shown in the figure. The median time from the onset of symptoms to the last positive stool specimen in the $\mathbf{4 9}$ patients was eight days (range two to 35). In children the median was eight days (range two to 35) and in adults it was seven days (range two to 19). Only three patients, all children, continued to shed oocysts after 20 days. The median time from the onset of symptoms to the first negative stool specimen in the 49 patients was 18 days (range nine to 50 ). In children the median was 19 days (range nine to 50 ) and in adults it was 17 days (range 11-28).

In 25 of the 49 patients it was possible to compare the duration of symptoms to the timing of their own stool specimens. In 19 (76\%) symptoms stopped between the range of the last positive and first negative stool specimen. In four $(16 \%)$ symptoms (mainly prolonged abdominal pain) continued after the first negative specimen and in two $(8 \%)$ shedding of oocysts continued after symptoms stopped.

\section{Discussion}

There are few published studies following up series of patients with cryptosporidiosis until their stools are clear. Those which do comprise small numbers and give widely varying results. There is also doubt about the association between symptoms and the shedding of oocysts. Both Hunt et al $^{8}$ and Stehr-Green et al ${ }^{9}$ found oocysts in the stool after the diarrhoea had ceased, but as we have shown diarrhoea is only one part of the symptomatology of cryptosporidiosis. ${ }^{2}$

In our study the taking and timing of stool specimens was influenced by the degree of illness of the patient, the practicing habits of the family doctors, the time it took for the infection control nurse to make contact with the family, and the compliance of the patient. A wide range of times after the onset of symptoms was therefore covered in the study and though in individual patients the interval between the last positive and first negative specimen was sometimes wide, the size of the group that we studied and the large number of stool specimens that we examined allows useful deductions to be made about the shedding of oocysts. We can confirm that the duration of shedding is long. We had no negative stools before nine days and most stools became negative between 11 and 23 days from the onset of symptoms. Few $(6 \%)$ of the patients continued to shed oocysts after 20 days. All of these patients in our series 
were children although others have shown adults with prolonged shedding of oocysts. ${ }^{9}$

Our study shows that when all symptoms are taken into account the shedding of oocysts is strongly correlated with the presence of symptoms and when symptoms persist after shedding ceases as in $16 \%$ of our patients the symptom is usually prolonged abdominal pain. It has been shown that mucosal changes occur in the intestine with cryptosporidiosis and this may be responsible for the prolonged symptoms in some patients. ${ }^{5}$

Using the onset of symptoms as the marker for the start of the illness is convenient. ${ }^{89}$ The incubation period representing the true onset of infection is clearly important in a study such as this. Blagburn and Current reporting an incident involving a researcher give an incubation period of five days, ${ }^{11}$ and Jokipii et al give a range of four to 12 days. ${ }^{12}$ Experimental work suggests that the incubation period may vary with the age of the patient. ${ }^{5}$ In our series we report an incubation period of two to 11 days, median seven. ${ }^{2}$ This work should make the counselling of patients about their illness easier and give a clearer idea of the likely period of isolation of children from nursery and school.

We thank Dr F E Russell, consultant microbiologist, Royal Alexandra Hospital, Paisley and his technical staff for allowing us access to their results and for their help, Dr Stella Gibson, community medicine specialist, Renfrew Priority Services Unit, for cooperation in the study, and to Miss Elizabeth Paton and Mrs Jean Still for secretarial help.

\section{References}

1 Bissenden JG. Cryptosporidium and diarrhoea. Br Med J 1986;293:287-8.

2 Shepherd RC, Sinha GP, Reed CL, Russell F. Cryptosporidium in the West of Scotland. Scot Med J (in press).

3 Isaacs D, Hunt GH, Phillips AD, Price EH, Raafat F, Walker-Smith JA. Cryptosporidiosis in immunocompetent children. J Clin Pathol 1985;38:76-81.

4 Malebranche R, Arnoux E, Guerin JM, et al. Acquired immune deficiency syndrome with severe gastrointestinal manifestations. Lancet 1983;ii:873-8.

5 Angus WA. Cryptosporidiosis in man, domestic animals and birds: a review. J R Soc Med 1983;76:62-70.

6 Bird RG, Smith MD. Cryptosporidiosis. J Pathol 1980;132: 217-33.

7 Current LW, Reese NC, Ernst JV, Bailey WS, Heyman MB, Weinstein WM. Human cryptosporidiosis in immunocompetent and immunodeficient persons. $N$ Engl $J$ Med 1983;308:1252-7.

8 Hunt D, Shannon R, Palmer SR, Jephcott AE. Cryptosporidiosis in an urban community. $\mathrm{Br}$ Med $J$ 1984;289:814-6.

9 Stehr-Green JK, McCaig LA, Remsen HM, Rains CS, Fox M, Juranek DD. Shedding of oocysts in immunocompetent individuals infected with Cryptosporidium. Am J Trop Med Hyg 1987;36:338-42.

10 Henriksen SA, Pohlenz JFL. Staining of Cryptosporidia by a modified Ziehl-Neelsen technique. Acta Vet Scand 1981;222:594-6.

11 Blagburn BL, Current WL. Accidental infection of a researcher with human Cryptosporidium. J Infect Dis 1983;148:772-3.

12 Jokipii L, Pohjola S, Jokipii AMM. Cryptosporidium: a frequent finding in patients with gastrointestinal symptoms. Lancet 1983;ii:358-61.

Requests for reprints to: Dr $\mathbf{R}$ Cameron Shepherd, Consultant Paediatrician, The Royal Alexandra Hospital, Corsebar Road, Paisley PA2 9PN, Scotland. 